\title{
THE RULED RESIDUE THEOREM FOR SIMPLE TRANSCENDENTAL EXTENSIONS OF VALUED FIELDS
}

\begin{abstract}
JACK OHM
Abstract. A proof is given for the Ruled Residue Conjecture, which asserts that if $v$ is a valuation of a simple transcendental field extension $K_{0}(x)$ and $v_{0}$ is the restriction of $v$ to $K_{0}$, then the residue field of $v$ is either ruled or algebraic over the residue field of $v_{0}$.
\end{abstract}

1. Introduction. Let $K_{0} \subset K=K_{0}(x)$ be fields with $x$ transcendental (abbreviated tr.) over $K_{0}$, let $v_{0}$ be a valuation of $K_{0}$ and $v$ be an extension of $v_{0}$ to $K$; and let $V_{0} \subset V, k_{0} \subset k$, and $G_{0} \subset G$ be the respective valuation rings, residue fields, and value groups.

We prove here the Ruled Residue Conjecture, which asserts that $k$ is either algebraic over $k_{0}$ or ruled over $k_{0}$. ("Ruled" means that there should be a field $k_{1}$ with $k_{0} \subset k_{1} \subset k$ and $k$ simple tr. over $k_{1}$; in the present setting such a $k_{1}$ is necessarily finite algebraic over $k_{0}$. Cf. [3].)

This conjecture was suggested by Nagata [2], who proved the case of discrete, finite rank $v_{0}$. The char. 0 case of the conjecture (i.e. char. $k_{0}=0$ ) was settled by the present author in [3], and Heinzer [1] subsequently improved this result to the case that $k_{0}$ is perfect. The proof of the full conjecture given below is completely elementary and direct, in contrast to the proofs of these special cases.

2. Additional notation. The notation ( $)^{*}$ will indicate image under the canonical homomorphism $V \rightarrow k$; and for $a \in K$ we shall write $a \rightarrow a^{*}$ to mean $a \in V$ and $a^{*}$ is the image of $a$ under the canonical homomorphism $V \rightarrow k$. Note that if $\xi \in V$ and $\xi \rightarrow \xi^{*}$ tr. over $k_{0}$, then $v(\xi)=0$ and $1 / \xi \rightarrow 1 / \xi^{*}$.

For any $f \neq 0$ in $K_{0}[x]$, define $\operatorname{deg} f$ to be $n$ if $f=a_{0}+a_{1} x+\cdots+a_{n} x^{n}$ with $a_{i} \in K_{0}$ and $a_{n} \neq 0$. Any $\xi \neq 0$ in $K_{0}(x)$ can be written $\xi=f / g$, where $f, g$ are nonzero, relatively prime elements of $K_{0}[x]$; and such $f, g$ are unique up to nonzero multiplies from $K_{0}$. We shall define $\operatorname{deg} \xi$ to be $\max \{\operatorname{deg} f, \operatorname{deg} g\}$. Note that if $\xi \in V$ and $\xi \rightarrow \xi^{*}$ tr. over $k_{0}$, then $\xi \notin K_{0}$ and hence $\operatorname{deg} \xi>0$.

Finally, if $k / k_{0}$ is not algebraic, then there exists $\xi \in V$ such that $\xi \rightarrow \xi^{*}$ tr. over $k_{0}$. In this case we shall let $S=\left\{\xi \in V \mid \xi \rightarrow \xi^{*}\right.$ tr. over $\left.k_{0}\right\}$ and $\min S=\{\xi \in S \mid$ $\operatorname{deg} \xi \leqslant \operatorname{deg} \zeta$ for all $\zeta \in S\}$.

Received by editors December 21, 1982.

1980 Mathematics Subject Classification. Primary 13A18, 12F20.

Key words and phrases. Valued fields, simple transcendental field extensions, ruled field extensions. 


\section{Proof of the theorem.}

3.1. Lemma. If there exists $\xi \in V$ of $\operatorname{deg} n$ such that $\xi \rightarrow \xi^{*}$ tr. over $k_{0}$, then there exists $\xi_{1} \in V$ such that $\xi_{1} \rightarrow \xi_{1}^{*}$ tr. over $k_{0}$ and $k_{0}\left(\xi_{1}^{*}\right)=k_{0}\left(\xi^{*}\right)$, and such that $\xi_{1}$ has the additional property that $\xi_{1}=f_{1} / g_{1}$, where $f_{1}, g_{1}$ are relatively prime elements of $K_{0}[x]$ with $\operatorname{deg} f_{1}=n, \operatorname{deg} g_{1}<n$.

Proof. Write $\xi=f / g$, where $f, g$ are relatively prime elements of $K_{0}[x]$. If $\operatorname{deg} g>\operatorname{deg} f$, then $\xi_{1}=1 / \xi$ works.

Thus, we may assume $\operatorname{deg} g=\operatorname{deg} f=n$; and we may then write $f=a g+r$, where $0 \neq a \in K_{0}, 0 \neq r \in K_{0}[x]$, and $\operatorname{deg} r<n$.

If $v(a) \geqslant 0$, then $r / g=(f / g)-a \in V$ and $(r / g)^{*}=(f / g)^{*}-a^{*}$ is tr. over $k_{0}$. Thus, $g / r=\xi_{1}$ works.

If $v(a)<0$, then $r / a f=(1 / a)-(g / f) \in V$ and $(r / a f)^{*}=(1 / a)^{*}-(g / f)^{*}$ is tr. over $k_{0}$. Thus, $a f / r=\xi_{1}$ works.

3.2. Lemma. Suppose $\xi=f / g$, where $f, 0 \neq g \in K_{0}[x]$ and $n=\operatorname{deg} f>\operatorname{deg} g$. Then

$$
K_{0}[x] \subset K_{0}[\xi]+K_{0}[\xi] x+\cdots+K_{0}[\xi] x^{n-1} ;
$$

or, equivalently, any $h \in K_{0}[x]$ may be written

$$
h=a_{m} \xi^{m}+a_{m-1} \xi^{m-1}+\cdots+a_{0},
$$

where $a_{i} \in K_{0}[x]$ and either $\operatorname{deg} a_{i}<n$ or $a_{i}=0$.

Proof. Let $M$ denote the $K_{0}[\xi]$-module, $K_{0}[\xi]+K_{0}[\xi] x+\cdots+K_{0}[\xi] x^{n-1}$. It suffices to see $x^{n} \in M$; for then it follows by induction on $s$ that $x^{n+s} \in M$ for all $s \geqslant 0$, and hence that $K_{0}[x] \subset M$.

Since $\operatorname{deg} f=n, x^{n}=a f+r$, where $0 \neq a \in K_{0}, r \in K_{0}[x]$, and either $\operatorname{deg} r<$ $\operatorname{deg} f$ or $r=0$. Then $x^{n}=(a g)(f / g)+r=(a g) \xi+r$, where $\operatorname{deg}(a g)=\operatorname{deg} g<n$ and either $\operatorname{deg} r<n$ or $r=0$.

3.3. Ruled Residue TheOrem. Suppose $k$ is not algebraic over $k_{0}$, and let $\xi \in \min S$. Then $k=k_{0}^{\prime}\left(\xi^{*}\right)$, where $k_{0}^{\prime}$ is the algebraic closure of $k_{0}$ in $k$.

Proof. The inclusion $\supset$ is immediate, so it remains to show $\subset$. By 3.1 we may assume $\xi=f / g$, where $\operatorname{deg} f=n>\operatorname{deg} g$. For any $\zeta \in V$, we may write, by 3.2 ,

$$
\zeta=\left(a_{m} \xi^{m}+a_{m-1} \xi^{m-1}+\cdots+a_{0}\right) /\left(b_{m} \xi^{m}+b_{m-1} \xi^{m-1}+\cdots+b_{0}\right),
$$

where $a_{i}, b_{i}$ are elements of $K_{0}[x]$ which are either of deg $<n$ or are 0 . Let $d$ be an element of least value from among $\left\{a_{i}, b_{i} \mid i=0, \ldots, m\right\}$, and let $\alpha_{i}=a_{i} / d, \beta_{i}=b_{i} / d$. Then

$$
\zeta=\left(\alpha_{m} \xi^{m}+\alpha_{m-1} \xi^{m-1}+\cdots+\alpha_{0}\right) /\left(\beta_{m} \xi^{m}+\beta_{m-1} \xi^{m-1}+\cdots+\beta_{0}\right),
$$

where now the coefficients $\alpha_{i}, \beta_{i}$ are elements of $K_{0}(x)$ which are either of deg $<n$ or are 0 . Moreover, $v\left(\alpha_{i}\right), v\left(\beta_{i}\right), i=0, \ldots, m$, are all $\geqslant 0$. We may therefore consider the equality

$$
\left(\beta_{m}^{*} \xi^{* m}+\cdots+\beta_{0}^{*}\right) \zeta^{*}=\alpha_{m}^{*} \xi^{* m}+\cdots+\alpha_{0}^{*}
$$


Since $\xi \in \min S$ and the $\alpha_{i}, \beta_{i}$ are either 0 or of $\operatorname{deg}<\operatorname{deg} \xi$, it follows that the $\alpha_{i}^{*}$, $\beta_{i}^{*}$ are all algebraic over $k_{0}$. But $\xi^{*}$ is tr. over $k_{0}$ and we know some $\alpha_{i}^{*}$ or $\beta_{i}^{*}$ is 1 , so some $\beta_{i}^{*}$ must be $\neq 0$. Therefore $\beta_{m}^{*} \xi^{* m}+\cdots+\beta_{0}^{*} \neq 0$, and hence

$$
\zeta^{*}=\left(\alpha_{m}^{*} \xi^{* m}+\cdots+\alpha_{0}^{*}\right) /\left(\beta_{m}^{*} \xi^{* m}+\cdots+\beta_{0}^{*}\right) \in k_{0}^{\prime}\left(\xi^{*}\right) .
$$

\section{REFERENCES}

1. W. Heinzer, Valuation rings and simple transcendental field extensions, J. Pure Appl. Algebra 26 (1982), 189-190.

2. M. Nagata, $A$ theorem on valuation rings and its applications, Nagoya Math. J. 29 (1967), 85-91.

3. J. Ohm, Simple transcendental extensions of valued fields, J. Math. Kyoto Univ. 22 (1982), 201-221.

Department of Mathematics, Louisiana State University, Baton Rouge, Louisiana 70803 